\title{
A risk score system based on DNA methylation levels and a nomogram survival model for lung squamous cell carcinoma
}

\author{
MING ZHANG, LIBING SUN, YI RU, SHASHA ZHANG, JUNJUN MIAO, \\ PENGDA GUO, JINGHUAN LV, FENG GUO and BIAO LIU
}

\begin{abstract}
Department of Oncology, The Affiliated Suzhou Municipal Hospital of Nanjing Medical University, Suzhou, Jiangsu 215001, P.R. China
\end{abstract}

Received September 14, 2019; Accepted January 30, 2020

DOI: $10.3892 /$ ijmm.2020.4590

\begin{abstract}
Lung squamous cell carcinoma (LSCC) is one of the primary types of non-small cell lung carcinoma, and patients with recurrent LSCC usually have a poor prognosis. The present study was conducted to build a risk score (RS) system for LSCC. Methylation data on LSCC (training set) and on head and neck squamous cell carcinoma (validation set 2) were obtained from The Cancer Genome Atlas database, and GSE39279 (validation set 1) was retrieved from the Gene Expression Omnibus database. Differentially methylated protein-coding genes (DMGs)/long non-coding RNAs (DM-lncRNAs) between recurrence-associated samples and nonrecurrence samples were screened out using the limma package, and their correlation analysis was conducted using the cor.test() function. Following identification of the optimal combinations of DMGs or DM-lncRNAs using the penalized package in R, RS systems were built, and the system with optimal performance was selected. Using the rms package, a nomogram survival model was then constructed. For the
\end{abstract}

Correspondence to: Dr Feng Guo or Dr Biao Liu, Department of Oncology, The Affiliated Suzhou Municipal Hospital of Nanjing Medical University, 16 Baita West Road, Suzhou, Jiangsu 215001, P.R. China

E-mail: guofeng27@suda.edu.cn

E-mail: liuyrew1234@hotmail.com

Abbreviations: AUC, area under the receiver operating characteristic curve; DEG, differentially expressed gene; DMG, differentially methylated gene; DM-lncRNA, differentially methylated lncRNA; FDR, false discovery rate; FC, fold change; HNSC, head and neck squamous cell carcinoma; KM, Kaplan-Meier analysis; lncRNA, long non-coding RNA; LSCC, lung squamous cell carcinoma; NSCLC, non-small cell lung cancer; LUAD, lung adenocarcinoma; PCC, Pearson correlation coefficient; RFS, recurrence-free survival; RS, risk score; ROC, receiver operating characteristic; TCGA, The Cancer Genome Atlas

Key words: lung squamous cell carcinoma, differential expression analysis, risk score system, nomogram survival model, pathway enrichment analysis differentially expressed genes (DEGs) between the high- and low-risk groups, pathway enrichment analysis was performed by Gene Set Enrichment Analysis. There were 335 DMGs and DM-lncRNAs in total. Following screening out of the top 10 genes (aldehyde dehydrogenase 7 family member A1, chromosome 8 open reading frame 48 , cytokine-like 1 , heat shock protein 90 alpha family class A member 1, isovaleryl-CoA dehydrogenase, phosphodiesterase 3A, PNMA family member 2, SAM domain, SH3 domain and nuclear localization signals 1 , thyroid hormone receptor interactor 13 and zinc finger protein 878) and 6 top lncRNAs, RS systems were constructed. According to Kaplan-Meier analysis, the DNA methylation level-based RS system exhibited the best performance. In combination with independent clinical prognostic factors, a nomogram survival model was built and successfully predicted patient survival. Furthermore, 820 DEGs between the high- and low-risk groups were identified, and 3 pathways were identified to be enriched in this gene set. The 10-DMG methylation level-based RS system and the nomogram survival model may be applied for predicting the outcomes of patients with LSCC.

\section{Introduction}

Lung cancer is a common cancer worldwide, with the second highest mortality rate among females and the highest mortality rate among males $(1,2)$. Non-small cell lung cancer (NSCLC) is the primary lung cancer type that accounts for $\sim 85 \%$ of lung cancer cases and includes the following common pathological subtypes: Lung squamous cell carcinoma (LSCC), lung adenocarcinoma (LUAD), and lung large cell carcinoma $(3,4)$. LSCC is more common among males, potentially due to smoking habits (5). LSCC has distinct clinical characteristics, and the prognosis of patients with recurrent LSCC is poor (6). Therefore, it is urgent to identify new molecular factors predictive of LSCC treatment outcomes.

Methylation changes in certain tumor-associated genes have been identified in previous studies examining tumorigenesis, suggesting that they are critical risk factors of tumorigenesis and molecular markers for early diagnosis (7). Promoter methylation of PR/SET domain 5 is significantly associated with lymph node metastasis and tumor differentiation status of LSCC; therefore, this gene is a candidate target for the 
diagnosis, prognosis and treatment of this cancer (8). The drought-repressed 4 gene has been proposed for methylation status analysis of LSCC cells, and its low expression correlates with a poor patient prognosis (9). Tripartite motif-containing $58 / \operatorname{cg} 26157385$ methylation is associated with expression of 8 prognosis-associated genes in LSCC, indicating its potential regulatory role in the progression of LSCC (10).

Long non-coding RNAs (lncRNAs) are a class of RNAs measuring $>200$ nucleotides and have pivotal functions in the progression of numerous cancer types (11). In addition, increasing numbers of $\operatorname{lncRNAs}$ with a prognostic value have been described, for example, urothelial cancer associated and long intergenic non-coding RNA-p21 (12-14). The lncRNAs cancer susceptibility 2 and surfactant associated 1 , pseudogene participate in the regulation of tumor suppressor genes and oncogenes during the formation of LSCC and may be used for the diagnosis, prognosis and targeted treatment of this disease (15). Several lncRNA combination signatures have been identified to predict LSCC prognosis $(16,17)$. In LUAD, DNA methylation has regulatory effects on the role of IncRNA (18); however, to the best of our knowledge, this type of regulation in LSCC has rarely been reported.

Despite these valuable data, the predictive performance of methylated DNA sequences or lncRNAs is rarely compared, and additional potential biomarkers should be investigated to improve cancer prognosis. In the present study, the methylation levels of DNA sequences and lncRNAs in patients with LSCC were compared, and the levels identified to be significantly correlated with LSCC prognosis were screened out. Furthermore, different risk score (RS) systems were built, and the one with the best predictive performance was selected.

\section{Materials and methods}

Data sources. Methylation data from patients with LSCC (downloaded on March 25, 2019; platform: Illumina Infinium Human Methylation 450 BeadChip) including 372 LSCC samples and 43 healthy tissue samples, were retrieved from The Cancer Genome Atlas (TCGA; https://cancergenome.nih. gov/) database. Following collation with clinical information, 293 LSCC samples with data recurrence were selected as the training set.

With 'lung cancer' and 'Homo sapiens' as key words, a search for methylation datasets was conducted in the Gene Expression Omnibus (GEO; http://www.ncbi.nlm.nih. gov/geo/) database (19) using the following criteria: i) Available histological data that could differentiate adenocarcinoma from squamous cell carcinoma; ii) total sample size was not $<150$; and iii) the clinical information associated with the LSCC samples contained data on actual future recurrence. Following this search, an eligible methylation dataset (accession no., GSE39279 (20); platform: Illumina HumanMethylation450 BeadChip; validation set 1) was selected and downloaded. There were 444 samples in the dataset, including 43 LSCC samples with data concerning future recurrence.

In addition, the methylation data on head and neck squamous cell carcinoma (HNSC), including 530 HNSC samples and 50 healthy tissue samples, were also retrieved from TCGA to investigate whether the predictive model for LSCC was applicable to other squamous carcinoma types. Following collation with the clinical information downloaded from TCGA simultaneously, 382 HNSC samples with information on future recurrence were selected as validation set 2 .

Differential methylation analysis. According to the data corresponding to probe locations and IDs provided in the downloaded annotation files that contained information about protein-coding genes and non-coding RNAs, like gene and lncRNA function, DNA location site and pathway information, corresponding lncRNAs and genes in the methylation data were annotated using the HUGO Gene Nomenclature Committee (http://www.genenames.org/) database (21), which contains records on 4,112 lncRNAs and 19,201 genes.

On the basis of the recurrence data, the LSCC samples in the training set were classified into recurrence-associated (the tumor recurred) and nonrecurrence (the tumor did not recur) groups. Using the limma package (22) (v.3.34.7, https://bioconductor. org/packages/release/bioc/html/limma.html) in the R software (23), differentially methylated genes (DMGs) between the recurrence-associated and nonrecurrence samples were identified. The thresholds for significance were defined as $\log _{2}$ fold change (FC) $>0.263$ and false discovery rate (FDR) $<0.05$. Following the addition of data on methylation levels of the DMGs in the training set, bidirectional hierarchical clustering was performed on the DMGs via the pheatmap package (v.1.0.8, https://cran.r-project. org/web/packages/pheatmap/index.html) in R (24).

Analysis of correlation between the methylation levels and expression levels of the DMGs. Data on the methylation levels and the matched expression levels of the DMGs were extracted, and their Pearson correlation coefficients (PCCs) were calculated using the cor.test() function (https://stat. ethz.ch/R-manual/R-devel/library/stats/html/cor.test.html) in $\mathrm{R}(25) . \mathrm{P}<0.05$ was set as the significance threshold. DMGs with negative PCCs were selected for further analyses.

Construction of RS systems. Using univariate and multivariate Cox regression analyses in $\mathrm{R}$ package survival (v3.1-8; https://www.rdocumentation.org/packages/survival/versions/3.1-8), differentially methylated lncRNAs (DM-lncRNAs) and DMGs significantly associated with overall survival and independent prognosis factors were next screened out. A log-rank $\mathrm{P}<0.05$ was selected as the cutoff criterion.

Based on the DMGs correlating with independent prognosis, an optimal gene combination and optimal lncRNA combination were identified using the LASSO Cox regression model (26) in penalized package in $\mathrm{R}$ (27) (v.0.9.50; https:/cran.r-project.org/web/packages/penalized/index.html). Optimal parameter 'lambda' in the model was calculated by the 1,000-fold cross-validation likelihood (cvl) method.

In combination with prognostic coefficients of the optimal DM-lncRNAs and DMGs, methylation status-based or methylation level-based RS systems were built.

For the optimal combinations, cutoff values of methylation levels of the optimal lncRNAs and genes were computed via the X-Tile Bio-Informatics Tool (https://medicine.yale. edu/lab/rimm/research/software.aspx) (28). The cutoff values were determined based on the following criterion: Monte Carlo $\mathrm{P}<0.05$. The status of each sample at an IncRNA or DNA methylation level was defined in accordance with the cutoff value of 
each IncRNA or gene. When the IncRNA or DNA methylation level was increased compared with the cutoff value, its status was set to 1.0. Otherwise, the status was set to 0 .

In combination with the regression coefficients of each optimal lncRNA or gene and their methylation status, a RS system was created, and the RS for each sample was calculated via the following formula:

$$
\text { Status } \mathrm{RS}=\sum \beta_{\mathrm{RNAn}} \times \text { Status }_{\mathrm{RNAn}} ;
$$

where $\beta$ and Status represent the regression coefficient of an lncRNA or gene and the methylation status variable, respectively. The status RS systems, based on the methylation status of the optimal genes and optimal lncRNAs, were constructed separately as two separate status RS systems.

RS systems based on the methylation levels of optimal IncRNAs or optimal genes were constructed. The formula for calculating the RS of each sample was as follows:

$$
\text { Methylation } \mathrm{RS}=\sum \beta_{\mathrm{RNAn}} \times \text { Methylation }_{\mathrm{RNAn}} \text {; }
$$

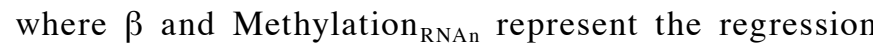
coefficient and methylation level of an lncRNA or gene, respectively. A methylation RS system based on the methylation levels of optimal genes and a methylation RS system based on the methylation levels of optimal lncRNAs were built separately.

Samples in the training set were subdivided into highand low-risk groups based on the median of the RSs of the two status RS systems and the two methylation RS systems. Using the Kaplan-Meier (KM) survival curve constructed by means of R package survival (29), associations between the prognosis and RS systems were analyzed. Concomitantly, the two validation sets were used to validate the results obtained. Finally, from the four RS systems generated, the system with optimum performance was selected through comparison of the parameters in the training set and the two validation sets.

Construction of a nomogram survival model. In the training set, independent prognostic factors were selected by univariate and multivariate Cox regression analyses via the survival package (29). The cutoff criterion was log-rank $\mathrm{P}<0.05$. According to the risk data determined by the $\mathrm{RS}$ system, the associations between the independent clinical prognostic factors and prognosis were then analyzed. Using the rms package (v.5.1-2, https://cran.r-project.org/web/packages/rms/index.html) (30) in R, a 5-year nomogram survival model involving the independent clinical prognostic factors was built.

Pathway enrichment analysis. RSs of the best RS system were used to classify the samples in the training set into high- and low-risk groups. Using the limma package (23), differential expression analysis of the high- and low-risk groups was performed to identify differentially expressed genes (DEGs) between the two risk groups. The DEGs were screened according to FDR $<0.05$ and $\log _{2} \mathrm{FCl}>0.263$ cut-off values Using Gene Set Enrichment Analysis (http://software.broadinstitute.org/gsea/index.jsp) (31), pathway enrichment analysis of the DEG set was conducted. The pathways with $\mathrm{P}<0.05$ were identified as significant.

\section{Results}

Differential-methylation analysis. On the basis of the annotation files, 1,028 lncRNAs and 15,544 genes were selected from the methylation dataset. According to the recurrence data, samples in the training set were subdivided into the recurrence-associated (78 LSCC samples from patients who later experienced cancer recurrence) and nonrecurrence (215 LSCC samples) groups. There were 335 DMGs and DM-lncRNAs between the recurrence-associated and nonrecurrence groups, including 27 DM-lncRNAs, 4 hypomethylated and 23 hypermethylated lncRNAs, and 308 DMGs, 139 hypomethylated and 169 hypermethylated genes (Fig. 1A). The bidirectional hierarchical clustering heatmap for the DMGs and GM-lncRNAs is presented in Fig. 1B.

Analysis of the correlation between the methylation levels and expression levels of the DMGs or DM-lncRNAs. As aforementioned, the matched methylation samples from the LSCC mRNA-seq samples were first selected, and the methylation and expression data on the DMGs and DM-lncRNAs were then extracted. The overall correlation between the methylation levels and expression levels of the DMGs/DM-lncRNAs were analyzed among the matched samples, and a significant negative correlation $\left(\mathrm{PCC}=-0.3857 ; \mathrm{P}=0.574 \times 10^{-13}\right.$ ) was identified (Fig. 2). A total of 181 DMGs and DM-lncRNAs with negative PCCs, including 25 DM-lncRNAs and 156 DMGs, were identified for subsequent analyses.

Construction of the RS system. Among the 181 DMGs and DM-lncRNAs, 105 were identified to be associated with prognosis, including 91 DMGs and 14 DM-lncRNAs, by univariate Cox regression analysis. Then, 16 DMGs and 6 DM-lncRNAs independently associated with prognosis were identified from the 105 DMGs/DM-lncRNAs using multivariate Cox regression analysis. According to the LASSO Cox regression model, 10 optimal DMGs, including aldehyde dehydrogenase 7 family member A1 (ALDH7A1), chromosome 8 open reading frame 48 (C8orf48), cytokine-like 1 ( $C Y T L 1)$, heat shock protein 90 alpha family class A member 1 (HSP90AAl), isovaleryl-CoA dehydrogenase (IVD), phosphodiesterase 3A (PDE3A), PNMA family member 2 (PNMA2), SAM domain, SH3 domain and nuclear localization signals 1 (SAMSN1), thyroid hormone receptor interactor $13(T R I P 13)$ and zinc finger protein 878 (ZNF878), and 6 optimal DM-lncRNAs, including ATXN8 opposite-strand lncRNA (ATXN8OS), brain cytoplasmic RNA 1 (BCYRN1), family with sequence similarity 138 member D (FAM138D), HLA complex group 11 (HCG11), MIR155 host gene (MIR155HG) and testis-specific transcript, Y-linked 13 (TTTY13) were finally identified in the training dataset (Fig. 3; Table I).

The cutoff values of the methylation levels of the lncRNAs and genes in the aforementioned optimal combinations were calculated (Table I). In combination with the regression coefficients of the optimal genes or lncRNAs, RS systems based on the methylation status of optimal genes or lncRNAs were constructed. The formulas were as follows: 

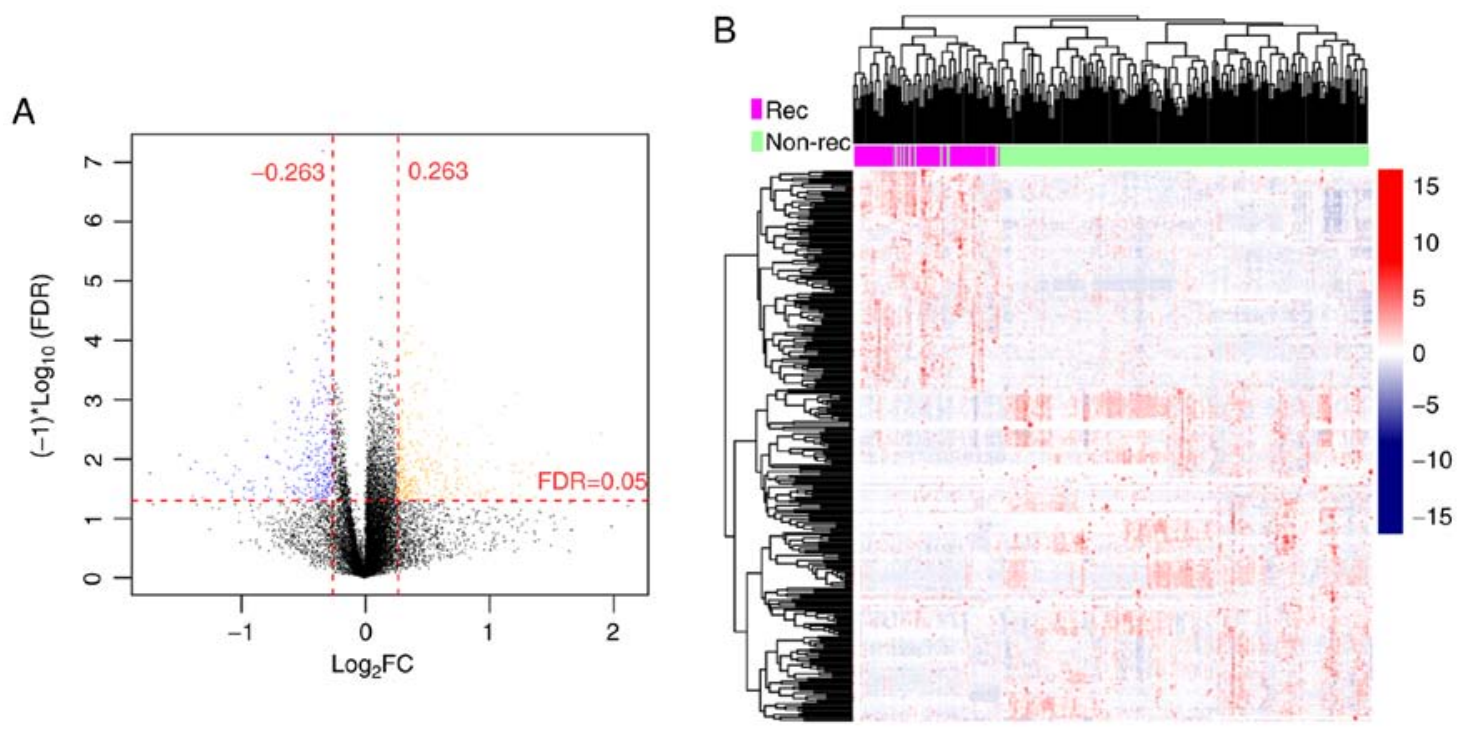

Figure 1. Identification of DMGs/DM-lncRNAs between recurrence-associated and nonrecurrence samples of LSCC tumors. (A) The volcano plot for the DMGs/DM-lncRNAs. The horizontal dashed line and the two vertical dashed lines represent FDR $<0.05$ and $\log _{2}$ FCl $>0.263$, respectively; blue and orange dots separately represent hypomethylated genes/lncRNAs and hypermethylated genes/lncRNAs. (B) The bidirectional hierarchical clustering heatmap for the DMGs/DM-lncRNAs. The purple and green strips represent recurrence-associated and nonrecurrence samples, respectively. DMGs, differentially methylated genes; DM-lncRNAs, differentially methylated lncRNAs; FDR, false discovery rate; FC, fold change; Rec, recurring; non-rec, non-recurring; lncRNAs, long non-coding RNAs.

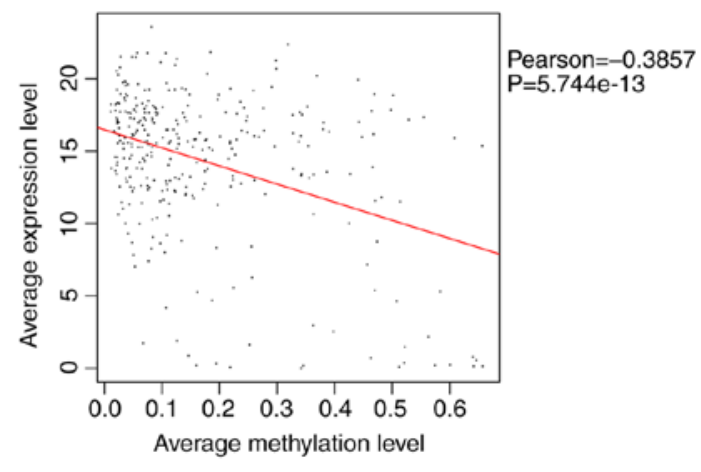

Figure 2. Scatter plot of the overall correlation between the methylation levels and expression levels of the differentially methylated genes/differentially methylated lncRNAs. The red line represents the trend line of the point distribution. lncRNAs, long non-coding RNAs.

Gene status $\mathrm{RS}=-1.1070 \mathrm{x}$ Status $\mathrm{ALDH7A1}-0.5721 \mathrm{x}$ Status $_{\mathrm{C} 8 \text { orf } 48}+0.4962 \times$ Status $_{\mathrm{CYTL} 1}+1.0118 \times$ Status $_{\mathrm{HSP90AA1}}+$ $0.5696 \times$ Status $_{\text {IVD }}+0.8992 \times$ Status $_{\text {PDE3A }}+0.0109 \times$ Status $_{\text {PNMA2 }}$ $+0.1803 \times$ Status $_{\mathrm{SAMSN} 1}+0.1612 \times$ Status $_{\text {TRIP13 }}-0.1688 \times$ Status $_{\text {ZNF878}}$; and lncRNA status $\mathrm{RS}=-1.2274 \times$ Status $_{\text {ATXN8Os }}$ +13.1984 x Status $_{\text {BCYRN } 1}+2.6252$ x Status $_{\mathrm{FAM} 138 \mathrm{D}}+2.9526 \mathrm{x}$ Status $_{\mathrm{HCG} 11}+1.2577$ x Status $_{\text {MIR } 155 \mathrm{HG}}+1.8308$ x Status $_{\mathrm{TTTY} 13}$.

In addition, RS systems based on the methylation levels of optimal genes or lncRNAs were built. The formulas for calculating the RSs were:

Gene methylation (methy) $\mathrm{RS}=-1.1070 \mathrm{x}$

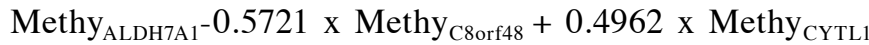
+1.0118 x Methy $_{\text {HSP90AA } 1}+0.5696$ x Methy $_{\text {IVD }}+0.8992$ x Methy $_{\mathrm{PDE} 3 \mathrm{~A}}+0.0109 \mathrm{x}$ Methy $\mathrm{PNMA}_{\mathrm{PN}}+0.1803 \mathrm{x}$ Methy $_{\mathrm{SAMSN} 1}+$ 0.1612 x Methy $_{\text {TRIP13 }}-0.1688 \times$ Methy $_{\text {ZNF878; }}$ and lncRNA methy $\mathrm{RS}=-1.2274 \times$ Methy $_{\mathrm{ATXN} 8 \mathrm{OS}}+13.1984 \mathrm{x} \mathrm{Methy}_{\mathrm{BCYRN} 1}+2.6252$ $\mathrm{x}_{\text {Methy }} \mathrm{FAM} 138 \mathrm{D}+2.9526 \mathrm{x}$ Methy $_{\mathrm{HCG} 11}+1.2577 \mathrm{x}_{\text {Methy }}$ MIR $155 \mathrm{HG}$ +1.8308 x Methy TTTY $13_{\text {. }}$.
According to the median RS values, samples in the training set, validation set 1 and validation set 2 were separately subdivided into high- and low-risk groups. The associations between prognosis and the results of RS systems were analyzed. The KM curves for the methylation status-based RS systems and those for the methylation level-based RS systems are presented in Figs. 4 and 5, respectively. These RS systems demonstrated high levels of specificity, with the area under the receiver operating characteristic curve $>0.6$ (Figs. 4 and 5), indicating accurate levels of risk prediction. Of all the systems generated, the RS system based on the methylation level of the 10 optimal DEGs exhibited the best predictive performance (Table II).

Construction of the nomogram survival model. Independent clinical factors associating with prognosis in the training set were identified by means of univariate and multivariate Cox regression analyses (Table III). For both the training and validation sets, pathological $\mathrm{T}$ stage, radiotherapy and DNA methylation level-based model RS status were selected as independent prognostic factors. The KM curves demonstrating the correlations between pathological T/radiotherapy and prognosis are presented in Fig. 6. The patients with low pathological $\mathrm{T}$ staging and those who underwent radiotherapy exhibited improved prognoses, which is consistent with the outcomes observed in clinical practice.

The 5-year nomogram survival model was built to further analyze the correlation between the 3 independent prognostic factors and prognosis; this model integrated various clinical indicators by means of the 'points' axis in the first row to predict the survival of patients corresponding to the samples (Fig. 7A). In addition, the predicted 5-year survival probability was compared to the actual 5-year survival probability, and a high level of consistency was observed (Fig. 7B). 
Table I. Genes and lncRNAs involved in the optimal combinations.

\begin{tabular}{|c|c|c|c|c|c|c|c|}
\hline Type & Gene & Locus & Coefficient & Hazard ratio & $95 \% \mathrm{CI}$ & $\mathrm{P}$-value & Cutoff \\
\hline \multirow[t]{10}{*}{ gene } & $A L D H 7 A 1$ & $\operatorname{cg} 26327732$ & -1.107 & 0.415 & $0.250-0.884$ & $2.350 \times 10^{-5}$ & 0.34 \\
\hline & C8orf48 & $\operatorname{cg} 24727311$ & -0.572 & 0.191 & $0.180-0.457$ & $4.610 \times 10^{-4}$ & 0.34 \\
\hline & CYTL1 & $\operatorname{cg} 17563034$ & 0.496 & 1.509 & $1.104-2.323$ & $3.051 \times 10^{-3}$ & 0.16 \\
\hline & HSP90AA1 & $\operatorname{cg} 23904247$ & 1.012 & 4.769 & $2.041-6.111$ & $1.080 \times 10^{-4}$ & 0.53 \\
\hline & $I V D$ & $\operatorname{cg} 27529930$ & 0.570 & 1.925 & $1.244-3.191$ & $3.500 \times 10^{-4}$ & 0.11 \\
\hline & $P D E 3 A$ & $\operatorname{cg} 26571814$ & 0.899 & 1.977 & $1.745-3.979$ & $9.310 \times 10^{-5}$ & 0.34 \\
\hline & PNMA2 & $\operatorname{cg} 26268277$ & 0.011 & 1.123 & $1.043-2.705$ & $7.295 \times 10^{-3}$ & 0.48 \\
\hline & SAMSN1 & $\operatorname{cg} 13951664$ & 0.180 & 1.208 & $1.079-2.546$ & $1.490 \times 10^{-4}$ & 0.25 \\
\hline & TRIP13 & $\operatorname{cg} 17510385$ & 0.161 & 1.33 & $1.038-2.691$ & $9.838 \times 10^{-3}$ & 0.65 \\
\hline & ZNF878 & $\operatorname{cg} 26626525$ & -0.169 & 0.597 & $0.336-0.906$ & $1.630 \times 10^{-3}$ & 0.06 \\
\hline \multirow[t]{6}{*}{$\operatorname{lncRNA}$} & ATXN8OS & $\operatorname{cg} 25514273$ & -1.227 & 0.312 & $0.088-0.704$ & $7.080 \times 10^{-3}$ & 0.24 \\
\hline & BCYRN1 & ch.X.1084981F & 13.198 & 5.399 & $1.028-10.38$ & $1.229 \times 10^{-2}$ & 0.04 \\
\hline & FAM138D & $\operatorname{cg} 26523196$ & 2.625 & 2.854 & $1.681-4.425$ & $9.330 \times 10^{-3}$ & 0.73 \\
\hline & $H C G 11$ & $\operatorname{cg} 27490387$ & 2.953 & 3.164 & $1.550-6.734$ & $2.010 \times 10^{-3}$ & 0.20 \\
\hline & MIR155HG & $\operatorname{cg} 23433889$ & 1.258 & 1.431 & $1.281-3.186$ & $3.341 \times 10^{-2}$ & 0.04 \\
\hline & $T T T Y 13$ & $\operatorname{cg} 25918849$ & 1.831 & 1.595 & $1.492-3.413$ & $6.200 \times 10^{-3}$ & 0.24 \\
\hline
\end{tabular}

lncRNA, long non-coding RNA; CI, confidence interval.

A
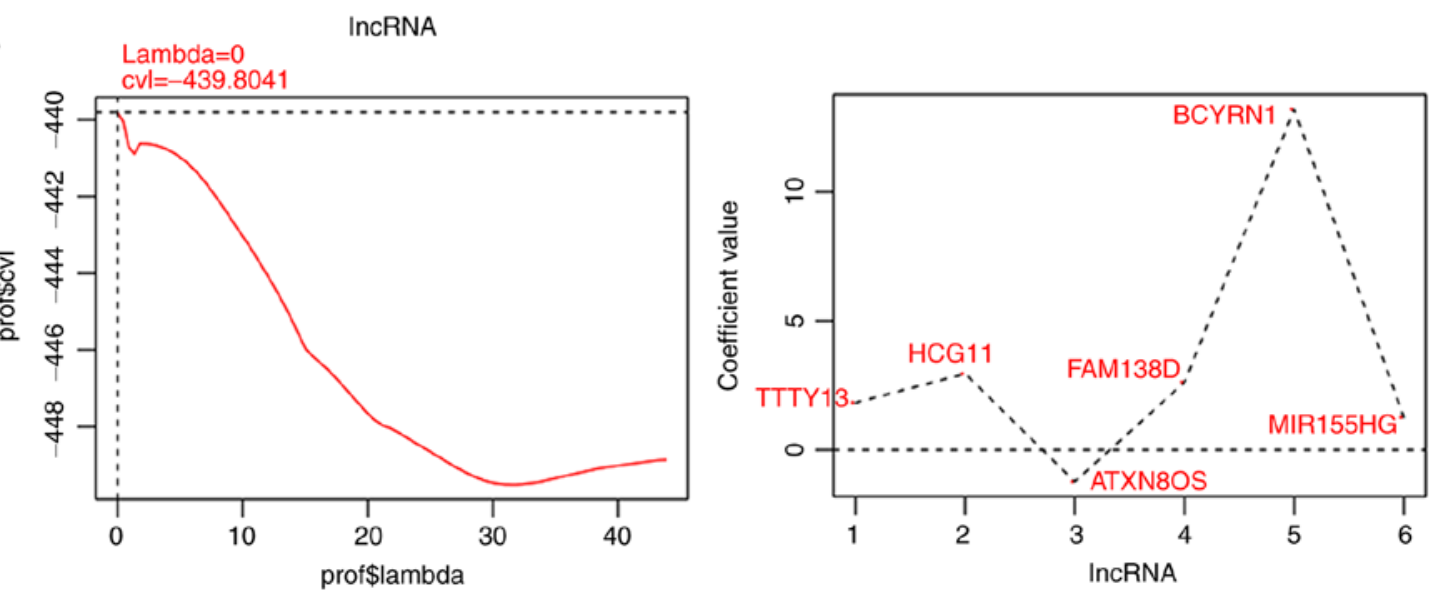

B
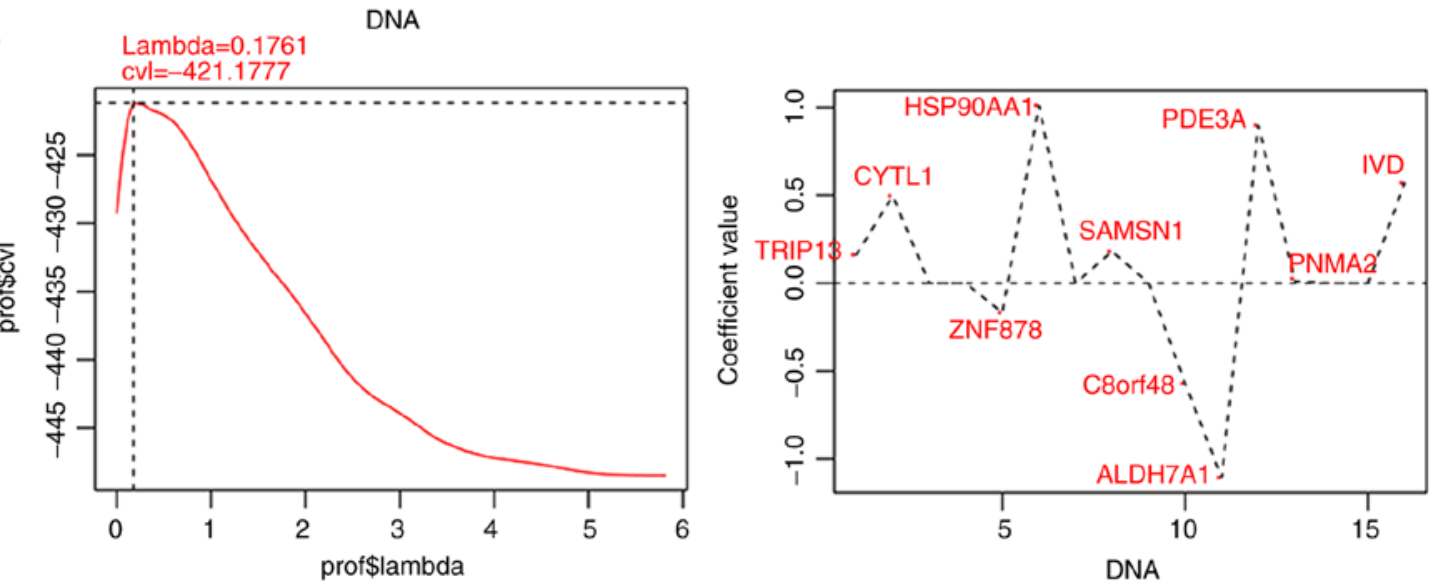

Figure 3. Graphs for selection of the 'lambda' parameter, and coefficient distribution diagrams of the optimal genes or lncRNAs. (A) The parameter selection diagram for lncRNAs. In the graph on the left, the horizontal and vertical axes represent 'lambda' and cross-validation likelihood (cvl) values, respectively; the intersection of the black dashed lines represents the 'lambda' value at the largest cvl value. The graph on the right demonstrates the coefficient distribution diagram of the optimal lncRNA. (B) The parameter selection diagram for genes. In the graph on the left, the horizontal and vertical axes respectively represent 'lambda' and cvl values; the intersection of the black dashed lines represents the 'lambda' value at the largest cvl value. Right: The graph on the right demonstrates the coefficient distribution diagram of the optimal gene. lncRNAs, long non-coding RNAs. 

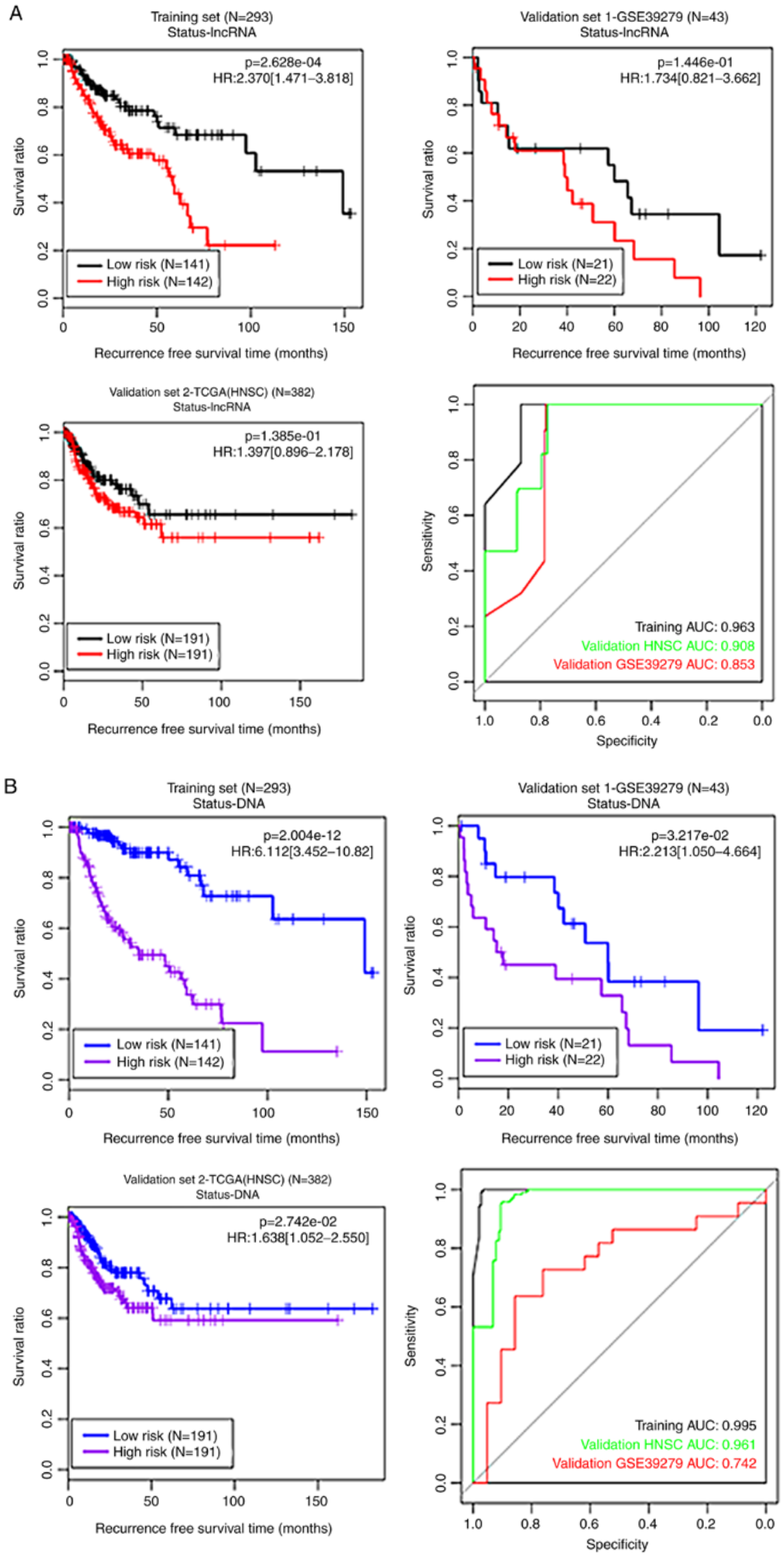

Figure 4. KM curves and ROC curves for the methylation status-based RS systems. (A) The KM curves (black and red curves respectively represent low- and high-risk groups) and the ROC curve (black, red and green lines represent the training set, validation set 1 and validation set 2, respectively) for the lncRNA methylation status-based RS system. (B) The KM curves (blue and purple curves respectively represent low- and high-risk groups) and the ROC curve (black, red and green lines represent the training set, validation set 1 and validation set 2, respectively) for the DNA methylation status-based RS system. KM, Kaplan-Meier; ROC, receiver operating characteristic; RS, risk score; TCGA, The Cancer Genome Atlas; HNSC, head and neck squamous cell carcinoma; AUC, area under the receiver operating characteristic curve; lncRNA, long non-coding RNA; HR, hazard ratio. 

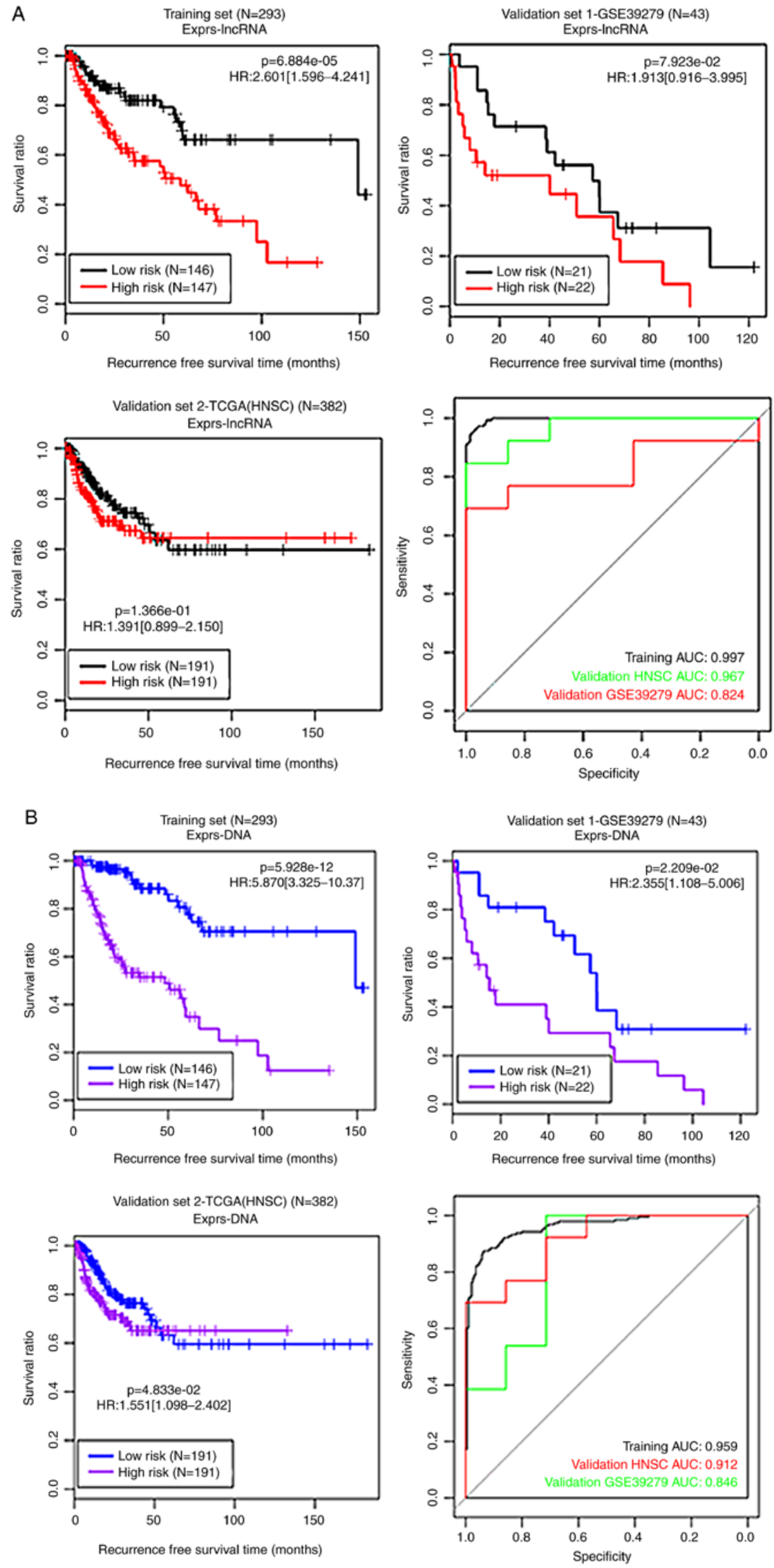

Figure 5. KM curves and ROC curves for the methylation level-based RS systems. (A) The KM curves (black and red curves represent low- and high-risk groups, respectively) and the ROC curve (black, red and green lines represent the training set, validation set 1 and validation set 2, respectively) for the long noncoding RNA (lncRNA) methylation level-based RS system. (B) The KM curves (blue and purple curves represent low- and high-risk groups, respectively) and the ROC curve (black, green and red lines represent the training set, validation set 1 and validation set 2, respectively) for the DNA methylation level-based RS system. KM, Kaplan-Meier; ROC, receiver operating characteristic; RS, risk score; TCGA, The Cancer Genome Atlas; HNSC, head and neck squamous cell carcinoma; AUC, area under the receiver operating characteristic curve; IncRNA, long non-coding RNA; HR, hazard ratio. 


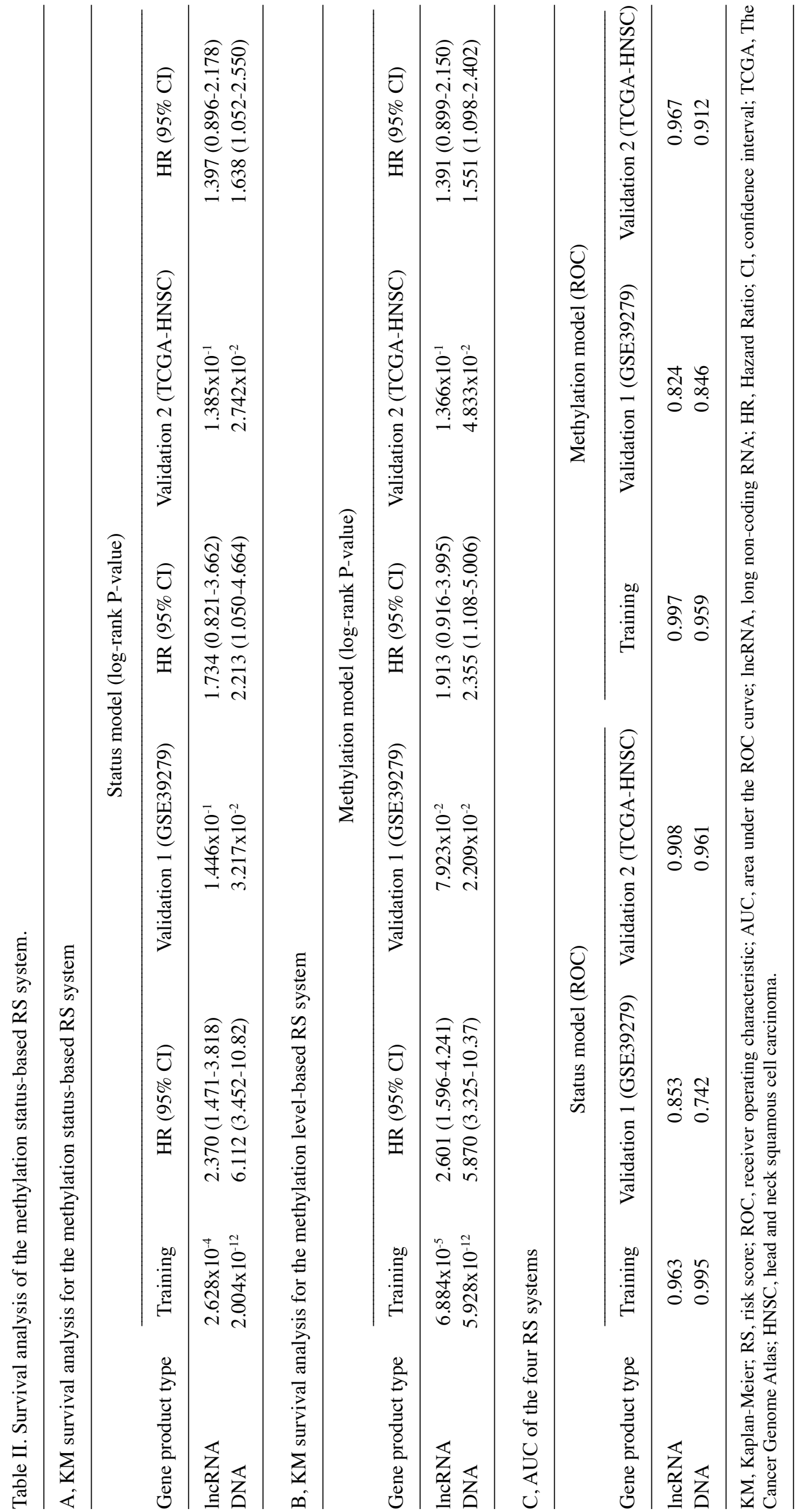




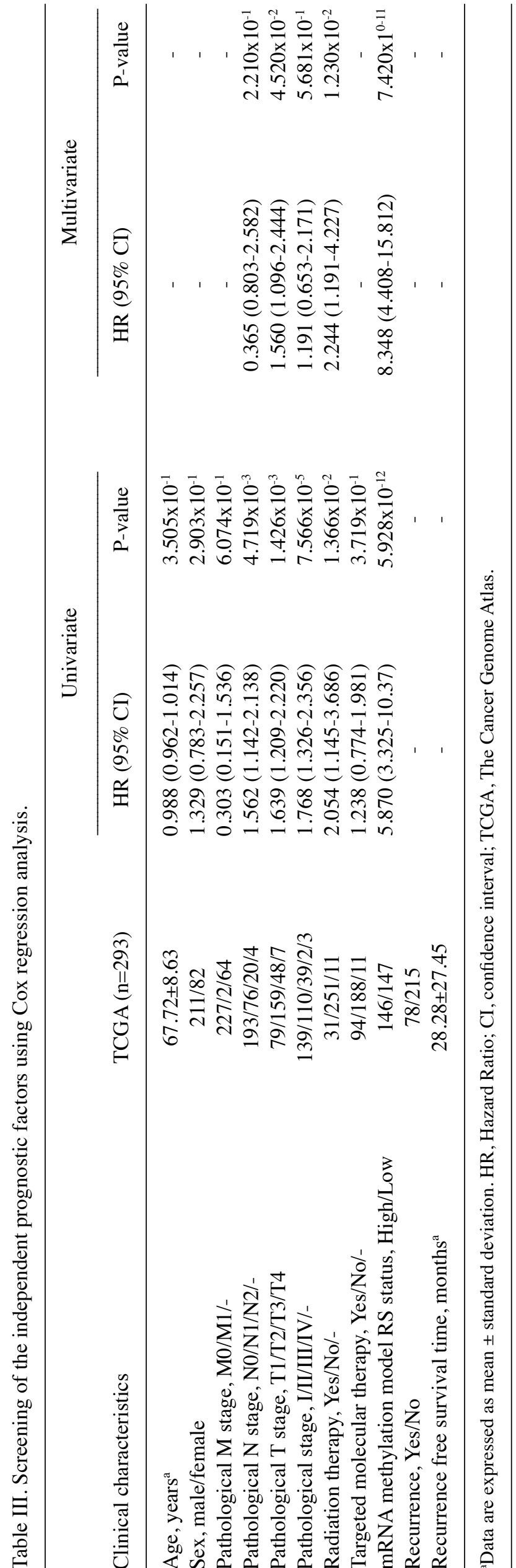

Pathway enrichment analysis. As aforementioned, following the addition of the RSs of the DNA methylation level-based RS system to the analysis, the samples in the training set were classified into either high- or low-risk groups. A total of 820 DEGs between the two groups were identified, including 412 upregulated genes and 408 downregulated genes. Following pathway enrichment analysis, 3 pathways were identified to be enriched in this set of DEGs: The calcium signaling pathway $(\mathrm{P}=0.040944)$; complement and coagulation cascades $(\mathrm{P}=0.0034123)$; and vascular smooth muscle contraction $(\mathrm{P}=0.0427049$; Table IV).

\section{Discussion}

In the present study, a total of 335 DMGs and DM-lncRNAs were identified between the recurrence-associated samples and nonrecurrence samples of tumor tissues from patients with LSCC. Among them, 16 DMGs and 6 DM-lncRNAs were significantly associated with independent prognosis, and 10 optimal DMGs (ALDH7A1, C8orf48, CYTL1, HSP90AAl, IVD, PDE3A, PNMA2, SAMSN1, TRIP13 and ZNF878) and 6 optimal DM-lncRNAs (ATXN8OS, BCYRN1, FAM138D, HCG11, MIR155HG and TTTY13) were used to construct the methylation status-based or methylation level-based RS systems. In the KM analysis, the 10-DMG methylation level-based RS system exhibited the best performance. The set of DEGs between high- and low-risk groups according to the RS system was identified to be enriched in 3 pathways: The calcium signaling pathway; complement and coagulation cascades; and vascular smooth muscle contraction. As the RS system based on the 10 DMGs exhibited an improved predictive performance compared with that based on the 6 lncRNAs, the present study focused on the functions of these 10 DMGs in LSCC.

Elevated expression of $A L D H 7 A 1$ in prostate cancer has been previously described, and was suggested to predict disease progression and metastasis (32), suggesting that alteration of the expression of this gene may control tumor progression. However, to the best of our knowledge, studies investigating the involvement of this gene in lung cancer are scarce. Low expression of $A L D H 7 A 1$ in tumors of surgically treated patients with NSCLC is associated with a decreased incidence of tumor recurrence, indicating that decreased expression of this gene may predict a good prognosis for these patients (33). Patients with LSCC with idiopathic pulmonary fibrosis indicate a significantly decreased methylation level of ALDH7Al compared with those without fibrosis (34). This observation suggests that methylation status of $A L D H 7 A 1$ may affect other clinical factors. In the present study, $A L D H 7 A 1$ was a component of the 10-DMG methylation level-based RS system that exhibited optimal performance; therefore, the methylation level of this gene may predict LSCC recurrence. Nevertheless, as the data concerning this gene in lung cancer are limited, the specific mechanisms of action of the ALDH7A1 protein and ALDH7Al DNA methylation in LSCC should be studied further.

CYTL1 has important roles in certain types of cancer and is regulated by DNA methylation in LSCC (35). The methylation patterns of $C Y T L 1$ are evidently different between early and late stages of LSCC, and hypermethylation is more common in the advanced stages (36). Although CYTL1 hypermethylation 

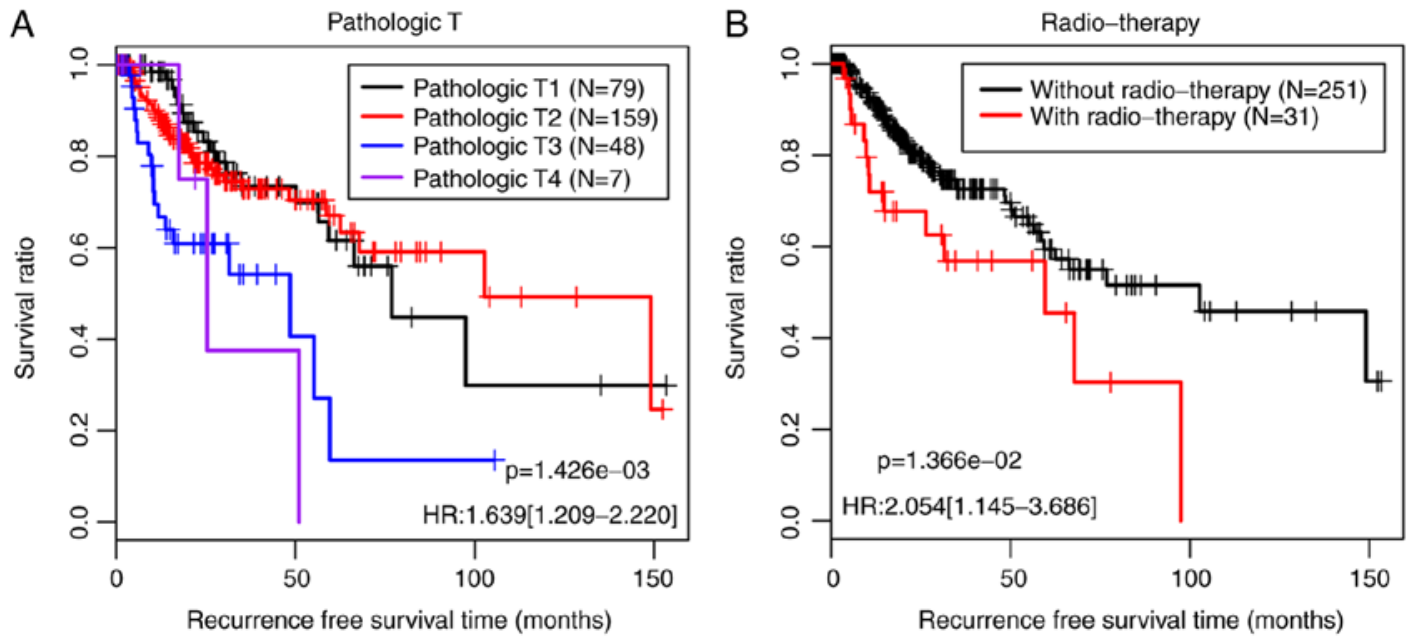

Figure 6. KM curves. (A) The KM curve revealing the correlation between pathological T and prognosis (black, red, blue and purple lines represent pathological T1, pathological T2, pathological T3 and pathological T4 stage, respectively); (B) The KM curve demonstrating the correlation between radiotherapy and prognosis (black and red lines represent patients without radiotherapy and those with radiotherapy, respectively). KM, Kaplan-Meier.

A
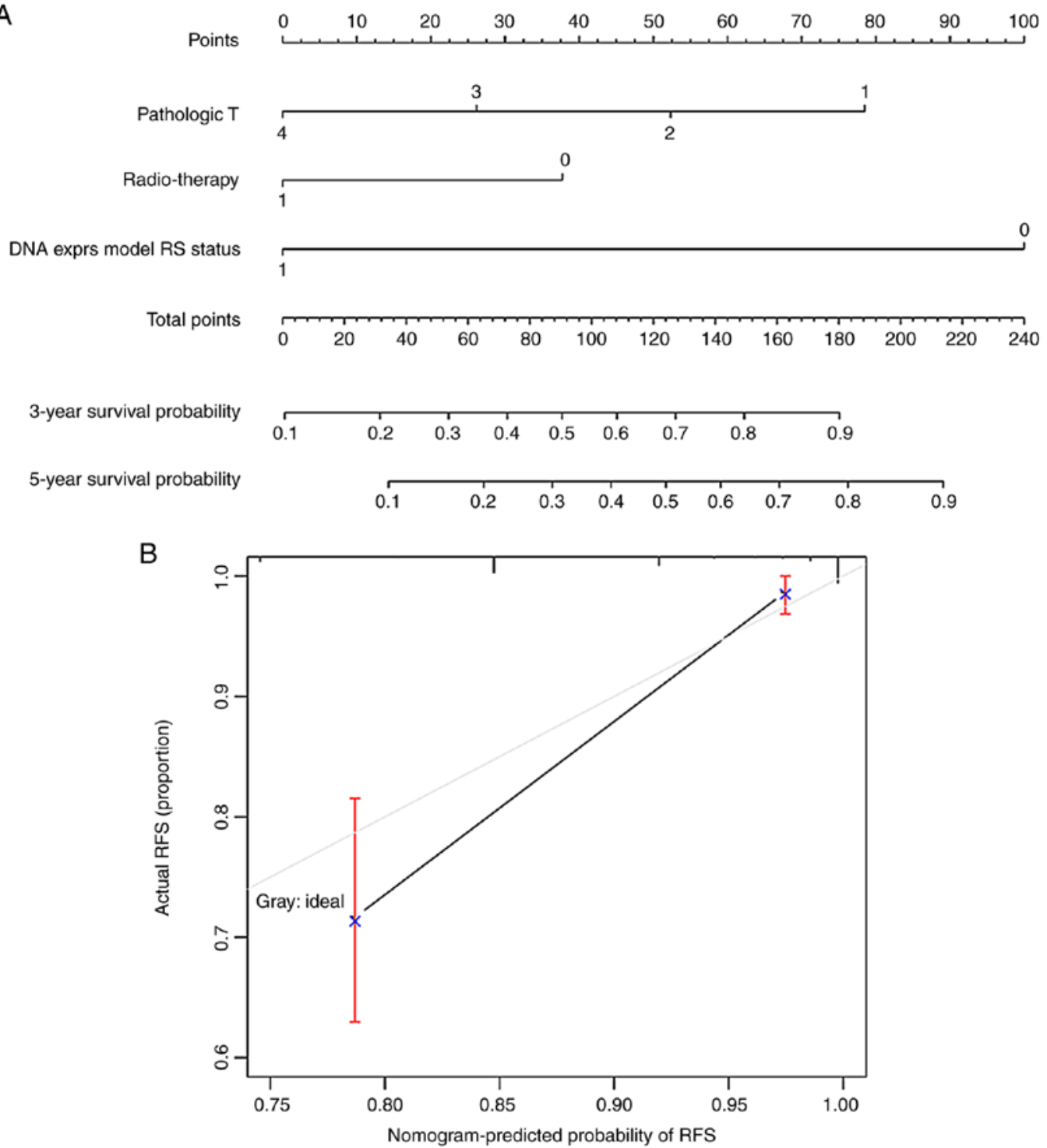

Figure 7. Nomogram survival model. (A) The nomogram survival model based on the independent prognostic factors. (B) The line graph indicating the consistency between the predicted 5-year survival probability and actual 5-year survival probability. The horizontal and vertical axes represent the predicted probability of RFS and the actual probability of RFS, respectively. The black line represents the trend line connecting the start time point and the 5-year time point. RFS, recurrence-free survival. 
Table IV. Pathways enriched for the differentially expressed genes.

\begin{tabular}{|c|c|c|c|c|c|}
\hline Term & Size & ES & NES & P-value & Genes \\
\hline $\begin{array}{l}\text { hsa04020: Calcium signaling } \\
\text { pathway }\end{array}$ & 10 & -0.396503 & -1.114227 & $4.094 \times 10^{-2}$ & $\begin{array}{l}E D N R B, H R H 1, C A L M L 3, P L N, L T B 4 R 2, \\
R Y R 1, B D K R B 2, C A L M L 5, I T P R 2, F 2 R\end{array}$ \\
\hline $\begin{array}{l}\text { hsa04610: Complement and } \\
\text { coagulation cascades }\end{array}$ & 10 & 0.5863498 & 2.0125692 & $3.412 \times 10^{-3}$ & $\begin{array}{l}C 3 A R 1, F 12, F G G, C 5, F 8, \text { SERPINA1, } \\
C 4 B P A, C 2, B D K R B 2, F 2 R\end{array}$ \\
\hline $\begin{array}{l}\text { hsa04270: Vascular smooth } \\
\text { muscle contraction }\end{array}$ & 7 & -0.537324 & -1.341176 & $4.270 \times 10^{-2}$ & $\begin{array}{l}\text { PTGIR, CALML3, CALML5, PRKG1, } \\
\text { PLA2G3, PPP1R14A, ITPR2 }\end{array}$ \\
\hline
\end{tabular}

ES, enrichment score; NES, normalized enrichment score.

does not affect the repression activity mediated by histone deacetylases (36), this repression activity may be subject to the regulation between $C Y T L 1$ hypermethylation and histone deacetylases, as the DNA methylation level is considered to affect the binding of a histone deacetylase to a promoter region $(36,37)$. CYTL1 is suggested to be a risk indicator of smoking-associated impairment of metabolic health, as it is hypomethylated and upregulated in non-lung tissues of smokers (38). According to the present study, the CYTL1 gene was often methylated in recurring LSCC tumors, suggesting that it may be a predictive factor of LSCC recurrence.

High expression levels of HSP90AAl, heat shock protein 90 alpha family class B member 1 and heat shock protein $90 \beta$ family member 1 are associated with adverse outcomes among patients with NSCLC, and therefore may serve as promising prognostic markers and therapeutic targets in NSCLC (39). HSP90AAl is differentially expressed between LUAD and LSCC, and therefore its expression profile may be used to distinguish the two subtypes (40). Nevertheless, data concerning HSP90AAl methylation or the effect of this gene on tumor recurrence are scarce. $P D E 3 A$ expression is low in chemoresistant NSCLC cells due to DNA hypermethylation, and high $P D E 3 A$ expression is associated with improved survival in patients with LUAD (41). According to the results of the present study, methylated $P D E 3 A$ may be associated with the prognosis of LSCC.

$P N M A 2$ is aberrantly expressed in various types of tumor in patients with paraneoplastic syndromes; therefore, PNMA2 may be implicated in tumorigenesis (42). PNMA2 has been demonstrated to be a tissue marker of small intestine neuroendocrine tumors, and Ma2 autoantibodies in the blood are a valuable biomarker for the diagnosis and prediction of tumor recurrence (43). Conversely, to the best of our knowledge, no studies on the methylation of this gene have been published, and this parameter may be a new prognostic indicator in LSCC, based on the results of the present study.

Downregulation of SAMSN1 is detectable in lung cancer cell lines and may be involved in the development of this disease (44). Elevated TRIP13 expression contributes to the progression of LUAD and may be a candidate biomarker or therapeutic target in LUAD (45). In lung cancer in Xuanwei, TRIP13, cAMP-responsive element-binding protein 3-like 4 and cyclin E2 exhibit concordant upregulation and frequent copy number gains, and have been proposed as potential oncogenes in the pathogenesis of lung cancer in this region (46). In addition, silencing of TRIP13 can suppress cell growth and metastasis of hepatocellular carcinoma by activating TGF- $\beta 1 /$ SMAD3 signaling (47). TRIP13 is 1 of the 7 hypomethylated genes in kidney renal cell carcinoma that have been suggested as a prognostic factor (48). Downregulation of ZNF878 has been suggested to be correlated with a poor prognosis of patients with LUAD (49). Although published data on SAMSN1, TRIP13, and ZNF878 methylation levels or their effects on LSCC are limited, when taken into consideration with the results of the present study, we hypothesize that these 3 genes may perform important functions in LSCC progression, and their methylation levels may help to determine an accurate prognosis of the disease.

Information on the participation of genes C8orf 48 and IVD in LSCC is scare. According to the results of the present study, their methylation may be a prognostic indicator of LSCC recurrence.

Despite the identification of the $10-\mathrm{DMG}$ signature that may predict the recurrence of LSCC, the present study contained certain limitations, such as the small sample size of the data retrieved from the database, and the lack of expression validation experiments. In addition, the clinical information of the LSCC samples in the training set was not compared to the validation sets, due to the difference in platforms. Nevertheless, this did preclude the predictive performance evaluation on risks of the identified clinical factors, as they could be distinguished from the AUC values. Further experiments are required to confirm the results.

In conclusion, 335 DMGs and DM-lncRNAs, including 27 DM-lncRNAs and 308 DMGs, were identified between recurrence-associated and nonrecurrence samples. The RS system based on the methylation levels of the 10 optimal DMGs (ALDH7A1, C8orf48, CYTL1, HSP90AA1, IVD, PDE3A, PNMA2, SAMSN1, TRIP13 and ZNF878) may help to predict the outcomes of patients with LSCC.

\section{Acknowledgements}

The results shown here are in whole or part based upon data generated by The Cancer Genome Atlas Research Network: https://www.cancer.gov/tcga.

\section{Funding}

The present study was supported by Suzhou Science and Technology Development Project (grant no. SYS2018085) and 
the Construction Project of Suzhou Clinical Medical Center (grant no. Szzx201506).

\section{Availability of data and materials}

All the data used in the present study are available from the Gene Expression Omnibus (http://www.ncbi.nlm.nih.gov/geo/; accession no., GSE39279) and The Cancer Genome Atlas public databases.

\section{Authors' contributions}

MZ, LS, YR, SZ, JM, PG and JL were responsible for data collection and statistical analysis. MZ, LS and YR participated in drafting and editing the manuscript. FG and BL were responsible for designing the work and reviewing the manuscript. BL obtained the funding. All authors read and approved the final manuscript.

\section{Ethics approval and consent to participate}

Not applicable.

\section{Patient consent for publication}

Not applicable.

\section{Competing interests}

The authors declare that they have no competing interests.

\section{References}

1. McGuire S: World cancer report 2014. Geneva, Switzerland: World health organization, international agency for research on cancer, WHO press, 2015. Adv Nutr 7: 418-419, 2016.

2. Hirsch FR and Bunn PA Jr: Progress in research on screening and genetics in lung cancer. Lancet Respir Med 2: 19-21, 2014.

3. Travis WD: Update on small cell carcinoma and its differentiation from squamous cell carcinoma and other non-small cell carcinomas. Mod Pathol 25 (Suppl 1): S18-S30, 2012.

4. Yoon SM, Shaikh T and Hallman M: Therapeutic management options for stage III non-small cell lung cancer. World J Clin Oncol 8: 1-20, 2017.

5. Tian S: Classification and survival prediction for early-stage lung adenocarcinoma and squamous cell carcinoma patients. Oncol Lett 14: 5464-5470, 2017.

6. Toyokawa G, Kozuma Y, Matsubara T, Haratake N, Takamori S, Akamine T, Takada K, Katsura M, Shimokawa M, Shoji F, et al Prognostic impact of controlling nutritional status score in resected lung squamous cell carcinoma. J Thorac Dis 9: 2942-2951, 2017.

7. Pfeifer G: Defining driver DNA methylation changes in human cancer. Int J Mol Sci 19: pii: E1166, 2018.

8. Tan SX, Hu RC, Tan YL, Liu JJ and Liu WE: Promoter methylation-mediated downregulation of PRDM5 contributes to the development of lung squamous cell carcinoma. Tumour Biol 35: 4509-4516, 2014

9. Wang W, Qi X and Wu M: Effect of DR4 promoter methylation on the TRAIL-induced apoptosis in lung squamous carcinoma cell. Oncol Rep 34: 2115-2125, 2015.

10. Zhang W, Cui Q, Qu W, Ding X, Jiang D and Liu H: TRIM58/cg26157385 methylation is associated with eight prognostic genes in lung squamous cell carcinoma. Oncol Rep 40 206-216, 2018.

11. Bhan A, Soleimani M and Mandal SS: Long noncoding RNA and cancer: A new paradigm. Cancer Res 77: 3965-3981, 2017.

12. Tong J, Ma X, Yu H and Yang J: SNHG15: A promising cancer-related long noncoding RNA. Cancer Manag Res 11: 5961-5969, 2019.
13. Yao F, Wang $\mathrm{Q}$ and $\mathrm{Wu} \mathrm{Q}$ : The prognostic value and mechanisms of lncRNA UCA1 in human cancer. Cancer Manag Res 11: 7685-7696, 2019.

14. Lu T, Wang Y, Chen D, Liu J and Jiao W: Potential clinical application of lncRNAs in non-small cell lung cancer. Onco Targets Ther 11: 8045-8052, 2018.

15. Huang GQ, Ke ZP, Hu HB and Gu B: Co-expression network analysis of long noncoding RNAs (IncRNAs) and cancer genes revealsSFTA1P and CASC2abnormalities in lung squamous cell carcinoma. Cancer Biol Ther 18: 115-122, 2017.

16. Li S, Teng Y, Yuan MJ, Ma TT, Ma J and Gao XJ: A seven long-noncoding RNA signature predicts prognosis of lung squamous cell carcinoma. Biomark Med 14: 53-63, 2020.

17. Huang G, Huang Q, Xie Z, Zhou H, Cao J, Shi L and Yang M: A nine-long non-coding RNA signature for prognosis prediction of patients with lung squamous cell carcinoma. Cancer Biomark 26: 239-247, 2019.

18. Li R, Yang YE, Yin YH, Zhang MY, Li H and Qu YQ: Methylation and transcriptome analysis reveal lung adenocarcinoma-specific diagnostic biomarkers. J Transl Med 17: 324, 2019.

19. Barrett T, Wilhite SE, Ledoux P, Evangelista C, Kim IF, Tomashevsky M, Marshall KA, Phillippy KH, Sherman PM, Holko M, et al: NCBI GEO: Archive for functional genomics data sets-update. Nucleic Acids Res 41 (Database Issue): D991-D995, 2013.

20. Sandoval J, Mendez-Gonzalez J, Nadal E, Chen G, Carmona FJ, Sayols S, Moran S, Heyn H, Vizoso M, Gomez A, et al: A prognostic DNA methylation signature for stage I non-small-cell lung cancer. J Clin Oncol 31: 4140-4147, 2013.

21. Yates B, Braschi B, Gray KA, Seal RL, Tweedie S and Bruford EA: Genenames.org: The HGNC and VGNC resources in 2017. Nucleic Acids Res 45 (D1): D619-D625, 2017.

22. Ritchie ME, Phipson B, Wu D, Hu Y, Law CW, Shi W and Smyth GK: limma powers differential expression analyses for RNA-sequencing and microarray studies. Nucleic Acids Res 43: e47, 2015.

23. Law CW, Alhamdoosh M, Su S, Dong X, Tian L, Smyth GK and Ritchie ME: RNA-seq analysis is easy as 1-2-3 with limma, Glimma and edgeR. F1000Res 5: 1408, 2016.

24. Wang L, Cao C, Ma Q, Zeng Q, Wang H, Cheng Z, Zhu G, Qi J, $\mathrm{Ma} \mathrm{H}$, Nian $\mathrm{H}$ and Wang Y: RNA-seq analyses of multiple meristems of soybean: Novel and alternative transcripts, evolutionary and functional implications. BMC Plant Biol 14: 169, 2014.

25. Kotthaus H, Korb I, Engel M and Marwedel P: Dynamic page sharing optimization for the R language. Acm Sigplan Notices 50: 79-90, 2014.

26. Kong S and Nan B: Non-asymptotic oracle inequalities for the high-dimensional Cox regression via lasso. Stat Sin 24: $25-42$, 2012.

27. Goeman JJ: L1 penalized estimation in the Cox proportional hazards model. Biom J 52: 70-84, 2010.

28. Camp RL, Marisa DF and Rimm DL: X-tile: A new bio-informatics tool for biomarker assessment and outcome-based cut-point optimization. Clin Cancer Res 10: 7252-7259, 2004

29. Liu XR, Pawitan Y and Clements MS: Generalized survival models for correlated time-to-event data. Stat Med 36: 4743-4762, 2017.

30. Steyerberg EW: FRANK E. HARRELL, Jr., Regression modeling strategies: With applications, to linear models, logistic and ordinal regression, and survival analysis, 2 nd ed. Heidelberg: Springer. Biometrics 72: 1006-1007, 2016.

31. Subramanian A, Tamayo P, Mootha VK, Mukherjee S, Ebert BL, Gillette MA, Paulovich A, Pomeroy SL, Golub TR, Lander ES and Mesirov JP: Gene set enrichment analysis: A knowledge-based approach for interpreting genome-wide expression profiles. Proc Natl Acad Sci USA 102: 15545-15550, 2005.

32. van den Hoogen C, van der Horst G, Cheung H, Buijs JT, Lippitt JM, Guzmán-Ramírez N, Hamdy FC, Eaton CL, Thalmann GN, Cecchini MG, et al: High aldehyde dehydrogenase activity identifies tumor-initiating and metastasis-initiating cells in human prostate cancer. Cancer Res 70: 5163-5173, 2010.

33. Giacalone NJ, Den RB, Eisenberg R, Chen H, Olson SJ, Massion PP, Carbone DP and Lu B: ALDH7A1 expression is associated with recurrence in patients with surgically resected non-small-cell lung carcinoma. Future Oncol 9: 737-745, 2013.

34. Hata A, Nakajima T, Matsusaka K, Fukuyo M, Morimoto J, Yamamoto T, Sakairi Y, Rahmutulla B, Ota S, Wada H, et al: A low DNA methylation epigenotype in lung squamous cell carcinoma and its association with idiopathic pulmonary fibrosis and poorer prognosis. Int J Cancer 146: 388-399, 2020. 
35. Zhu S, Kuek V, Bennett S, Xu H, Rosen V and Xu J: Protein Cyt11: Its role in chondrogenesis, cartilage homeostasis, and disease. Cell Mol Life Sci 76: 3515-3523, 2019.

36. Kwon YJ, Lee SJ, Koh JS, Kim SH, Lee HW, Kang MC, Bae JB, Kim YJ and Park JH: Genome-wide analysis of DNA methylation and the gene expression change in lung cancer. J Thorac Oncol 7: 20-33, 2012.

37. Kim SN, Kim NH, Lee W, Seo DW and Kim YK: Histone deacetylase inhibitor induction of P-glycoprotein transcription requires both histone deacetylase 1 dissociation and recruitment of CAAT/enhancer binding protein beta and pCAF to the promoter region. Mol Cancer Res 7: 735-744, 2009.

38. Tsai PC, Glastonbury CA, Eliot MN, Bollepalli S, Yet I, Castillo-Fernandez JE, Carnero-Montoro E, Hardiman T, Martin TC, Vickers A, et al: Smoking induces coordinated DNA methylation and gene expression changes in adipose tissue with consequences for metabolic health. Clin Epigenetics 10: 126, 2018.

39. Liu K, Kang M, Li J, Qin W and Wang R: Prognostic value of the mRNA expression of members of the HSP90 family in non-smal cell lung cancer. Exp Ther Med 17: 2657-2665, 2019.

40. Lu C, Chen H, Shan Z and Yang L: Identification of differentially expressed genes between lung adenocarcinoma and lung squamous cell carcinoma by gene expression profiling. Mol Med Rep 14: 1483-1490, 2016.

41. Tian FM, Zhong CY, Wang XN and Meng Y: PDE3A is hypermethylated in cisplatin resistant non-small cell lung cancer cells and is a modulator of chemotherapy response. Eur Rev Med Pharmacol Sci 21: 2635-2641, 2017.

42. Lee YH, Pang SW and Tan KO: PNMA2 mediates heterodimeric interactions and antagonizes chemo-sensitizing activities mediated by members of PNMA family. Biochem Biophys Res Commun 473: 224-229, 2016.

43. Cui T, Hurtig M, Elgue G, Li SC, Veronesi G, Essaghir A, Demoulin JB, Pelosi G, Alimohammadi M, Öberg K and Giandomenico V: Paraneoplastic antigen Ma2 autoantibodies as specific blood biomarkers for detection of early recurrence of small intestine neuroendocrine tumors. PLoS One 5: e16010, 2010
44. Yamada H, Yanagisawa K, Tokumaru S, Taguchi A, Nimura Y, Osada H, Nagino M and Takahashi T: Detailed characterization of a homozygously deleted region corresponding to a candidate tumor suppressor locus at 21q11-21 in human lung cancer. Genes Chromosomes Cancer 47: 810-818, 2008.

45. Li W, Zhang G, Li X, Wang X, Li Q, Hong L, Shen Y, Zhao C, Gong X, Chen Y and Zhou J: Thyroid hormone receptor interactor 13 (TRIP13) overexpression associated with tumor progression and poor prognosis in lung adenocarcinoma. Biochem Biophys Res Commun 499: 416-424, 2018.

46. Zhang Y, Xue Q, Pan G, Meng QH, Tuo X, Cai X, Chen Z, Li Y, Huang T, Duan X and Duan Y: Integrated analysis of genome-wide copy number alterations and gene expression profiling of lung cancer in Xuanwei, China. PLoS One 12: e0169098, 2017.

47. Yao J, Zhang X, Li J, Zhao D, Gao B, Zhou H, Gao S and Zhang L: Silencing TRIP13 inhibits cell growth and metastasis of hepatocellular carcinoma by activating of TGF- $\beta 1 / \mathrm{smad} 3$. Cancer Cell Int 18: 208, 2018.

48. Hu F, Zeng W and Liu X: A gene signature of survival prediction for kidney renal cell carcinoma by multi-omic data analysis. Int J Mol Sci 20: pii: E5720, 2019.

49. Liu $\mathrm{H}$ and Zhao $\mathrm{H}$ : Prognosis related miRNAs, DNA methylation, and epigenetic interactions in lung adenocarcinoma. Neoplasma 66: 487-493, 2019.

This work is licensed under a Creative Commons Attribution-NonCommercial-NoDerivatives 4.0 International (CC BY-NC-ND 4.0) License. 